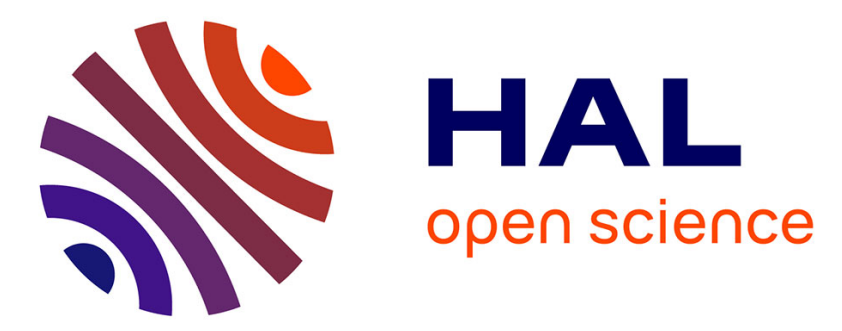

\title{
SECOND-ORDER ELASTICITY AND FIRST-ORDER FREDERIKS TRANSITIONS IN NEMATIC LIQUID CRYSTALS
}

\author{
H. Hinov, A. Derzhanski
}

\section{- To cite this version:}

H. Hinov, A. Derzhanski. SECOND-ORDER ELASTICITY AND FIRST-ORDER FREDERIKS TRANSITIONS IN NEMATIC LIQUID CRYSTALS. Journal de Physique Colloques, 1979, 40 (C3), pp.C3-505-C3-509. 10.1051/jphyscol:19793101 • jpa-00218702

HAL Id: jpa-00218702

https://hal.science/jpa-00218702

Submitted on 1 Jan 1979

HAL is a multi-disciplinary open access archive for the deposit and dissemination of scientific research documents, whether they are published or not. The documents may come from teaching and research institutions in France or abroad, or from public or private research centers.
L'archive ouverte pluridisciplinaire HAL, est destinée au dépôt et à la diffusion de documents scientifiques de niveau recherche, publiés ou non, émanant des établissements d'enseignement et de recherche français ou étrangers, des laboratoires publics ou privés. 


\title{
SECOND-ORDER ELASTICITY AND FIRST-ORDER FREDERIKS TRANSITIONS IN NEMATIC LIQUID CRYSTALS
}

\author{
H. P. HINOV and A. I. DERZHANSKI \\ Institute of Solid State Physics, Bulgarian Academy of Sciences, Sofia 1113, Bulgaria
}

\begin{abstract}
Résumé. - On a étudié théoriquement l'influence de l'élasticité de second ordre $\left(K_{13} \neq 0\right)$ sur les déformations électrostatiques dans des couches homéotropes, planaires et tordues nématiques. L'anisotropie diélectrique peut être positive ou négative. Pour certaines valeurs des énergies de surface anisotrope $K_{13} \lesssim W_{s} d \lesssim 3 K_{13}$ ( $d$ est l'épaisseur des couches) les déformations $0-\pi / 2$ apparaissent soudainement dans les couches homéotropes nématiques $\left(K_{13}>0\right)$ ou dans les couches planaires et tordues nématiques $\left(K_{13}<0\right)$. L'effet d'hystérésis peut être réglé à l'aide d'énergie d'ancrage.
\end{abstract}

\begin{abstract}
The influence of second order elasticity $\left(K_{13} \neq 0\right)$ on the electrostatic deformations in homeotropic, planar and twisted nematic layers with positive or negative dielectric anisotropy is considered theoretically. For some values of the anisotropic interfacial energies $K_{13} \lesssim W_{\mathrm{s}} d \lesssim 3 K_{13}$ ( $d$ is the thickness of the layer) the deformations $0-\pi / 2$ start with a jump in homeotropic nematic layers $\left(K_{13}>0\right)$ or in planar or twisted nematic layers $\left(K_{13}<0\right)$. The effect of the hysteresis may be regulated with the aid of the surface anchoring energy.
\end{abstract}

1. Introduction. - The static deformations in nematic liquid layers with Rapini's [1] type surface anchoring start at infinitely small angles (Frederiks transitions of a second order). De Gennes and E. Dubois-Violette [2] emphasized that the order of the transition depends significantly on the form of the surface energy. They pointed out that the surface energy may have a sharp minimum at a certain value of $\dot{\theta}_{0}$ and a flat plateau elsewhere. These assumptions were confirmed independently by Hinov [3] and Piesik and Koswig [4]. They pointed out that such a type of surface energies create Frederiks transition of the first order (the static deformations start with a jump at finite values of the angle).

Another way to give rise to Frederiks transition of the first order was noted by Deuling and Helfrich [5] for homeotropic nematic liquid crystal layers with a large anisotropic conductivity.

The exact threshold conditions obtained by Derzhanski and Hinov [6] for polar flexoelectric deformations including second order elasticity demonstrated steeper Frederiks transition at some critical values of the surface energy.

All these effects indicate a hysteresis in the switching characteristic which is in agreement with Rondelez' [7] assumptions.
Second-order elasticity may create a first order Frederiks transition in homeotropic, planar and twisted nematic layers for some values of the surface energy (assumed in this paper to be of the Rapini type).

We have calculated the necessary range for the surface energy providing a first order Frederiks transition $0-\pi / 2$ for homeotropic, planar and twisted nematic layers. This case, in our opinion, is most advantageous for improved optical performance and multiplexing capability. The obtained theoretically thresholds $0-\pi / 2$ (for increasing electric fields) and $\pi / 2-0$ (for decreasing electric fields) are different and the hysteresis depends on the values of $W_{\mathrm{s}}$ and on the second-order elasticity coefficient $K_{13}$.

The first order Frederiks transition $\theta-\pi / 2$ (for increasing electric fields) and $\pi / 2-\theta$ (for decreasing electric fields) is more complex and will be considered in another paper.

2. Theory. - Let us consider the free energy of the nematic layer (planar, homeotropic and twisted) subjected to an electric field and written in an invariant vector form by Nehring and Saupe [8] :

$$
\begin{aligned}
& H_{\mathbf{E}}=\int_{V} \mathrm{~d} V\left\{(1 / 2)\left[K_{11}^{\prime}(\operatorname{div} \mathbf{n})^{2}+K_{22}(\mathbf{n} \cdot \operatorname{rot} \mathbf{n})^{2}+K_{\mathbf{3} 3}^{\prime}(\mathbf{n} \times \operatorname{rot} \mathbf{n})^{2}\right]+\right. \\
& \left.\quad+K_{13} \operatorname{div}(\mathbf{n} \operatorname{div} \mathbf{n})-(1 / 8 \pi)\left[\varepsilon_{\|, \mathbf{L}}(\mathbf{E} \cdot \mathbf{n})^{2}+\varepsilon_{\perp, \|}(\mathbf{E} \times \mathbf{n})^{2}\right]\right\}+(1 / 2) W_{\mathbf{s}}(\mathbf{v n})^{2} .
\end{aligned}
$$


It represents a sum of the elastic energy (with an accuracy to the second derivatives of the director $\mathbf{n}$ with respect to the coordinates), the approximate dielectric energy accurate to a large extent for NLC's with small dielectric anisotropy $\left(\Delta \varepsilon / \varepsilon_{\perp, \|}\right)<1$ and the surface energy. The following physical quantities in CGSE units are introduced in [1] $: K_{11}^{\prime}, K_{22}$ and $K_{33}^{\prime}$ are elastic coefficient of splay, twist and bend respectively, $\varepsilon_{\|}$and $\varepsilon_{\perp}$ are the permitivites of the LC along the liquid crystal optical axis and along the normal, $K_{13}$ is the second-order elastic coefficient, $v$ is the normal to the nematic layer surface.

The introduction of a rectangular coordinate system $O X Y Z$ is most convenient for the problem thus set where the $X$ axis coincides with the lower interface surface, $Z$ is the normal and $Y$ brings the system to the right orientation.

The electric field increasing from zero to higher values and decreasing from the complete orientation of the LC to zero is denoted by $\uparrow \downarrow E$ respectively.

2.1 CASE A (HOMEOTROPIC, PLANAR LAYERs). When the components of the vector field $n_{x}=\sin \theta$, $n_{z}=\cos \theta$ and $E_{z}=E$ (for homeotropic layers) are substituted in the expression for the free energy [1] it is transformed into a functional of the deformation angle $\theta(z)$. Varying this functional with respect to $\theta(z) / H_{\mathrm{E}}[\theta(z)]$ is transformed into a functional of the inverse function $z(\theta)$ and is varied as a functional with mivable boundaries gives the differential equation describing the deformations in the layer :

$$
f(\theta)\left(\frac{\mathrm{d} \theta}{\mathrm{d} z}\right)^{2}+(|\Delta \varepsilon| / 4 \pi) E^{2} \sin ^{2} \theta=C
$$

where

$$
f(\theta)=K_{\mathbf{3} 3}^{\prime} \cos ^{2} \theta+K_{11}^{\prime} \sin ^{2} \theta
$$

and the rather complicated boundary conditions for the values of $\theta(z)$ at the two interfacial surfaces :

$$
\begin{aligned}
f(\theta)\left(\frac{\mathrm{d} \theta}{\mathrm{d} z}\right)-K_{13} \cos 2 \theta\left(\frac{\mathrm{d} \theta}{\mathrm{d} z}\right)- & \\
- & K_{13} \sin \theta \cos \theta\left(\frac{\mathrm{d} \theta}{\mathrm{d} z}\right)^{-1} \frac{d}{\mathrm{~d} z}\left(\frac{\mathrm{d} \theta}{\mathrm{d} z}\right) \\
& \pm W_{\mathrm{s}} \sin \theta \cos \theta=0 \quad z=d, 0
\end{aligned}
$$

where $\mathrm{d} \theta / \mathrm{d} z \neq 0, d$ is the thickness of the LC layer. The presence of the second derivative in the boun- dary conditions is a result of the elastic surface energy due to second-order elasticity $\left(K_{13} \neq 0\right)$. This energy introduces essentially nonlinear terms in the boundary conditions and complicates the problem very much. Another characteristic feature of these boundary conditions is their manifest dependence on the electric field $E$.

A quadratic equation for $\mathrm{d} \theta / \mathrm{d} z$ is obtained for the two surfaces (see Derzhanski and Hinov [6]) from (2) and (3) :

$$
a\left(\frac{\mathrm{d} \theta}{\mathrm{d} z}\right)^{2}+b \frac{\mathrm{d} \theta}{\mathrm{d} z}+c=0 \quad z=d, 0
$$

where

$$
\begin{aligned}
& a=f(\theta)-K_{13} \cos 2 \theta-K_{13}\left(K_{33}^{\prime}-K_{11}^{\prime}\right) \times \\
& \times[f(\theta)]^{-1} \sin ^{2} \theta \cos ^{2} \theta \\
& b= \pm W_{\mathrm{s} 2,1} \sin \theta \cos \theta \\
& c=K_{13}[f(\theta)]^{-1} \sin ^{2} \theta \cos ^{2} \theta(|\Delta \varepsilon| / 4 \pi) E^{2} \text {. }
\end{aligned}
$$

The following important conclusions can be drawn from (4) : the value of the derivative of the deformation angle $\theta(z)$ at the surfaces depends not only on the anisotropic elastic energy $W_{\mathrm{s}}$ but on the dielectric energy as well $(|\Delta \varepsilon| / 4 \pi) E^{2}$. The sign of the coefficient $b$ is of great importance and determines the type of deformation in the LC layer under investigation. It is evident that it is determined from the sign of the anisotropic interfacial energy $W_{\mathrm{s}}$ and dictates the sign of $\mathrm{d} \theta / \mathrm{d} z$ with respect to that of $\theta(z)$ at the interfaces. Physically this means that :

1. Deformations with a maximum in the bulk of the LC layer are established when the bulk torques are deforming and the surface ones are stabilizing with respect to the initial state of the LC.

2. Deformations with a minimum in the bulk of the LC layer are established when the bulk torques are stabilizing and the surface ones are deforming with respect to the initial state of the LC.

The deformation with a maximum in the LC layer is realized in the problem under consideration regarding less of the value of the applied electric field. Deformations with a minimum in the bulk of the LC layer can not be established in the case of a deforming action of the bulk and surface torques (a large electric field) and therefore LC is completely oriented along the applied electric field :

$$
z=\left\{\begin{array}{c}
\operatorname{sgn} \theta\left[(|\Delta \varepsilon| / 4 \pi) E^{2}\right]^{-1 / 2} \int_{\theta_{\mathrm{s}}}^{\theta}\left[\frac{f(\theta)}{\sin ^{2} \theta_{\mathrm{m}}-\sin ^{2} \theta}\right]^{1 / 2} \mathrm{~d} \theta, \frac{d}{\mathrm{~d} z} \theta^{2}>0 \\
\operatorname{sgn} \theta\left[(|\Delta \varepsilon| / 4 \pi) E^{2}\right]^{-1 / 2} \int_{\theta_{\mathrm{s}}}^{\theta_{\mathrm{m}}}\left[\frac{f(\theta)}{\sin ^{2} \theta_{\mathrm{m}}-\sin ^{2} \theta}\right]^{1 / 2} \mathrm{~d} \theta \\
-\int_{\theta_{\mathrm{m}}}^{\theta}\left[\frac{f(\theta)}{\sin ^{2} \theta_{\mathrm{m}}-\sin ^{2} \theta}\right]^{1 / 2} \mathrm{~d} \theta, \quad \frac{d}{\mathrm{~d} z} \theta^{2}<0
\end{array}\right.
$$


where

$$
\sin ^{2} \theta_{\mathrm{m}}=\frac{f(\theta)\left(\frac{\mathrm{d} \theta}{\mathrm{d} z}\right)^{2}}{(|\Delta \varepsilon| / 4 \pi) E^{2}}+\sin ^{2} \theta \quad z=0, d
$$

and

$$
\frac{\mathrm{d} \theta}{\mathrm{d} z}= \pm\left\{\frac{W_{\mathrm{s}}}{2 g(\theta)}+\left[\left(\frac{W_{\mathrm{s}}}{2 g(\theta)}\right)^{2}-\frac{K_{13}(|\Delta \varepsilon| / 4 \pi) E^{2}}{f(\theta) g(\theta)}\right]^{1 / 2}\right\} \sin \theta \cos \theta
$$

where

$$
g(\theta)=f(\theta)-K_{13} \cos 2 \theta-K_{13}\left(K_{33}^{\prime}-K_{11}^{\prime}\right)[f(\theta)]^{-1} \sin ^{2} \theta \cos ^{2} \theta .
$$

The following transcendental equation for the dimensionless parameter $U / U_{0}\left(U_{0}\right.$ is the threshold for fixed vanishing boundary conditions of the homeotropic nematic layer) was obtained following Barratt and Jenkins [9] and Leslie [10] after changing the variables $\sin \lambda=\sin \theta / \sin \theta_{\mathbf{m}}$ and the vanishing conditions $\theta(z=0, d), \theta_{\mathrm{m}} \rightarrow 0$ :

where

$$
(\pi / 2) U_{\mathrm{t}}^{\prime} / U_{0}=\operatorname{arctg} X_{\mathrm{s} \uparrow \boldsymbol{E}}
$$

$$
X_{\mathrm{s} \uparrow E}=\frac{W_{\mathrm{s}} d}{2 \pi\left(K_{33}^{\prime}-K_{13}\right)} \frac{U_{0}}{U_{\mathrm{t}}^{\prime}}+\left[\left(\frac{W_{\mathrm{s}} d}{2 \pi\left(K_{33}^{\prime}-K_{13}\right)} \frac{U_{0}}{U_{\mathrm{t}}^{\prime}}\right)^{2}-\frac{K_{13}}{K_{33}^{\prime}-K_{13}}\right]^{1 / 2} .
$$

The transcendental equation for the aligning field (complete orientation of the LC) (see also Nehring, Kmetz and Scheffer [11]) was obtained after changing the variables $c h=\cos \theta / \cos \theta_{\mathrm{m}}$ and the vanishing conditions $\theta(z=0, d)$ and $\theta_{\mathrm{m}} \rightarrow \pi / 2:\left(U_{0}^{*}\right.$ is for a fixed planar layer $)$

$$
(n / 2) U_{\mathrm{t}}^{\prime \prime} / U_{0}^{*}=\operatorname{Arth} X_{\mathrm{s} \downarrow E}
$$

where

$$
X_{\mathrm{s} \downarrow E}=\frac{W_{\mathrm{s}} d}{2 \pi\left(K_{11}^{\prime}+K_{13}\right)} \frac{U_{0}^{*}}{U_{\mathrm{t}}^{\prime \prime}}+\left[\left(\frac{W_{\mathrm{s}} d}{2 \pi\left(K_{11}^{\prime}+K_{13}\right)} \frac{U_{0}^{*}}{U_{\mathrm{t}}^{\prime \prime}}\right)^{2}-\frac{K_{13}}{K_{11}^{\prime}+K_{13}}\right]^{1 / 2} .
$$

The type of the Frederiks transition depends on the sign of $K_{13}$ and the values of the surface energy. Frederiks transition of a first order is realized for the following values of $W_{\mathrm{s}} d ;\left(K_{13}>0\right)$

$$
\begin{aligned}
& W_{\mathrm{s}} d \leqslant 4\left\{\operatorname{arctg}\left[\frac{K_{13}}{K_{33}^{\prime}-K_{13}}\right]^{1 / 2}\right\}\left[\frac{K_{13}}{K_{33}^{\prime}-K_{13}}\right]^{1 / 2}\left(K_{33}^{\prime}-K_{13}\right) \\
& W_{\mathrm{s}} d \geqslant 4\left\{\operatorname{Arth}\left[\frac{K_{13}}{K_{11}^{\prime}+K_{13}}\right]^{1 / 2}\right\}\left[\frac{K_{13}}{K_{11}^{\prime}+K_{13}}\right]^{1 / 2}\left(K_{11}^{\prime}+K_{13}\right)
\end{aligned}
$$

obtained after an exclusiour of the parameters $U_{\mathrm{t}}^{\prime} / U_{0}$ and $U_{\mathrm{t}}^{\prime \prime} / U_{0}^{*}$ from the relations

$$
(\pi / 2)\left(U_{\mathrm{t}}^{\prime} / U_{0}\right)=\frac{W_{\mathrm{s}} d}{2 \pi\left(K_{33}^{\prime}-K_{13}\right)} \frac{U_{0}}{U_{\mathrm{t}}^{\prime}}=\left(\frac{K_{13}}{K_{33}^{\prime}-K_{13}}\right)^{1 / 2}
$$

and

$$
(\pi / 2)\left(U_{\mathrm{t}}^{\prime \prime} / U_{0}^{*}\right)=\frac{W_{\mathrm{s}} d}{2 \pi\left(K_{11}^{\prime}+K_{13}\right)} \frac{U_{0}^{*}}{U_{\mathrm{t}}^{\prime \prime}}=\left(\frac{K_{13}}{K_{11}^{\prime}+K_{13}}\right)^{1 / 2}
$$

respectively.

The threshold voltages are respectively:

$$
\begin{gathered}
U_{\mathrm{t}}^{\prime} / U_{0} \leqslant(2 / \pi) \operatorname{arctg}\left[\frac{K_{13}}{K_{33}^{\prime}-K_{13}}\right]^{1 / 2} \\
U_{\mathrm{t}}^{\prime \prime} / U_{0}^{*} \geqslant(2 / \pi) \operatorname{Arth}\left[\frac{K_{13}}{K_{11}^{\prime}+K_{13}}\right]^{1 / 2} .
\end{gathered}
$$

It is clear from (6) that when the surface parameter $W_{\mathrm{s}} d$ obeys the inequality (9) the finite deformations $\theta$ at the surfaces do not stabilize the LC layer and a Frederiks transition $0-\pi / 2$ is realized. The hysteresis in the switching properties may be calculated (at a given value of $W_{\mathrm{s}} d$ for the range pointed out in (9)) from (7) and (8). 
This hysteresis is at a maximum for the largest value of the parameter $W_{\mathrm{s}} d$ in the pointed interval (9) :

$$
\frac{U_{t}^{\prime}}{U_{0}}-\frac{U_{t}^{\prime \prime}}{U_{0}^{*}}=(2 / \pi)\left[\operatorname{arctg}\left(\frac{K_{13}}{K_{33}^{\prime}-K_{13}}\right)^{1 / 2}-\operatorname{Arth}\left(\frac{K_{13}}{K_{11}^{\prime}+K_{13}}\right)^{1 / 2}\right]
$$

and disappears for the smallest values of $W_{\mathrm{s}} d$ :

$$
W_{\mathrm{s}} d=4\left(K_{11}^{\prime}+K_{13}\right)\left(\frac{K_{13}}{K_{11}^{\prime}+K_{13}}\right)^{1 / 2} \operatorname{Arth}\left(\frac{K_{13}}{K_{11}^{\prime}+K_{13}}\right)^{1 / 2} \text {. }
$$

For smaller values of the surface energy the LC is unstable at infinitely weak voltages. The higher order elasticity however may be come into a play in this case which can stabilize the LC below an unknown threshold.

When the parameter $W_{\mathrm{s}} d$ is slightly larger the situation is more complex. Then in the LC a Frederiks transition of a first order $\theta-\pi / 2$ (where $\theta$ depends on the value of $W_{\mathrm{s}} d$ ) is realized. A second order Frederiks transition exists in the LC when the saturation voltage is larger than the threshold one. It is easy to show after expansion of the functions in (7) and (8) that the inequality $W_{\mathrm{s}} d>\pi K_{13}$ is sufficient for an establishment of stable deformations $0 \leqslant \theta \leqslant \pi / 2$ in the LC layer.

In this manner the second order elasticity with an appropriate sign of the elastic coefficient $K_{13}$ leads to a first order Frederiks transition $0-\pi / 2$ for weak anchoring $\left(W_{\mathrm{s}} d \sim K_{13}\right)$.

This is valid and for a planar nematic layer ( $-K_{13}$ and $K_{11}^{\prime}$ must be substituted for $K_{13}$ and $K_{33}^{\prime}$, the sign of $K_{13}$ is a positive) as well.

2.2 CASE B (TWISTED LAYER). - Substituting the components $n_{x}=\cos \theta \cos \varphi, n_{y}=\sin \theta \sin \varphi, n_{z}=\sin \theta$ and $E_{z}=E$ in the free energy expression (1) transforms the latter into a functional of the deformation angles $\theta(z)$ and $\varphi(z)$. Varying this functional with respect to $\theta(z)$ and $\varphi(z)$ gives the differential equations describing the deformations in the layer :

$$
\begin{aligned}
& \frac{d}{\mathrm{~d} z}\left\{\frac{d}{\mathrm{~d} z}\left(1+\alpha \sin ^{2} \theta\right) \cos ^{2} \theta\right\}=0 \\
& \frac{d}{\mathrm{~d} z}\left\{\frac{\mathrm{d} \theta}{\mathrm{d} z}\left(1+k \sin ^{2} \theta\right)\right\}=k \sin \theta \cos \theta\left(\frac{\mathrm{d} \theta}{\mathrm{d} z}\right)^{2}+\frac{1+k}{1+\alpha}\left(\frac{\mathrm{d} \varphi}{\mathrm{d} z}\right)^{2} \times \\
& \times\left\{\alpha \sin \theta \cos ^{3} \theta-\left(1+\alpha \sin ^{2} \theta\right) \sin \theta \cos \theta\right\}-\left(|\Delta \varepsilon| / 4 \pi K_{11}^{\prime}\right) E^{2} \sin \theta \cos \theta
\end{aligned}
$$

where

$$
k=\frac{K_{33}^{\prime}-K_{11}^{\prime}}{K_{11}^{\prime}}, \quad \alpha=\frac{K_{33}^{\prime}-K_{22}}{K_{22}}, \quad \frac{\varepsilon_{\|}-\varepsilon_{\perp}}{\varepsilon_{\perp}}<1 .
$$

For small dielectric anisotropy the solutions $\theta(z)$ and $\varphi(z)$ can be written in the following form :

$$
\begin{gathered}
\varphi(z)=\beta \int_{0}^{\theta(z)} \frac{\left(1+k \sin ^{2} \theta\right)^{1 / 2}}{g(\theta) \cos ^{2} \theta\left(1+\alpha \sin ^{2} \theta\right)} \mathrm{d} \theta \\
(\pi / 2)\left(U / U_{0}\right)=\int_{0}^{\theta} \frac{\left(1+k \sin ^{2} \theta\right)}{g(\theta)} \mathrm{d} \theta \\
U_{0}=\pi\left(4 \pi K_{11}^{\prime} / \Delta \varepsilon\right)^{1 / 2}
\end{gathered}
$$

where

$$
g(\theta)=\sin ^{2} \theta_{\mathrm{m}}-\sin ^{2} \theta-\beta^{2} \frac{1+k}{1+\alpha}\left[\frac{1}{\left(1+\sin ^{2} \theta\right) \cos ^{2} \theta}-\frac{1}{\left(1+\sin ^{2} \theta_{\mathrm{m}}\right) \cos ^{2} \theta_{\mathrm{m}}}\right]^{1 / 2}
$$

Around the threshold

$$
g(\theta) \sim\left[\left(1+\beta^{2} \frac{1+k}{1+\alpha}(1-\alpha)\right)^{1 / 2}\left(\sin ^{2} \theta_{m}-\sin ^{2} \theta\right)\right]
$$

The following threshold transcendental equations (12), (13) for the dimensionless parameter $U / U_{0}\left(U_{0}\right.$ is the threshold for fixed vanishing boundary conditions for the planar nematic layer) were obtained from (3), (14) and (15) with $z=d$ after changing the variables at $\theta(z=0, d), \theta_{\mathrm{m}} \rightarrow 0, \pi / 2$ respectively : (see the case A) 


$$
\begin{gathered}
\operatorname{tg}(\pi / 2)\left\{\left(U_{\mathrm{t}}^{\prime} / U_{0}\right)^{2}+\frac{2 K_{22}-K_{33}^{\prime}}{\left.K_{11}^{\prime}\left(\varphi_{\mathrm{m}} / \pi\right)^{2}\right\}^{1 / 2}=}\right. \\
=1 / 2 \frac{W_{\mathrm{s}} d}{\pi\left(K_{11}^{\prime}+K_{13}\right)\left\{\left(U_{\mathrm{t}}^{\prime} / U_{0}\right)^{2}+\frac{2 K_{22}-K_{33}^{\prime}}{K_{11}^{\prime}}\left(\varphi_{\mathrm{m}} / \pi\right)^{2}\right\}^{1 / 2}} \\
+\left\{\left[\frac{W_{\mathrm{s}} d}{2 \pi\left(K_{11}^{\prime}+K_{13}\right)\left\{\left(U_{\mathrm{t}}^{\prime} / U_{0}\right)^{2}+\frac{2 K_{22}-K_{33}^{\prime}}{K_{11}^{\prime}}\left(\varphi_{\mathrm{m}} / \pi\right)^{2}\right\}^{1 / 2}}\right]^{1 / 2} \frac{K_{13}}{K_{11}^{\prime}+K_{13}}\right\}^{1 / 2} \\
(\pi / 2)\left(U_{\mathrm{t}}^{\prime \prime} / U_{0}\right)=\operatorname{Arth} X_{\mathrm{s} \backslash E}
\end{gathered}
$$

and

$$
X_{\mathrm{s} \backslash E}=\frac{W_{\mathrm{s}} d}{2 \pi\left(K_{33}^{\prime}-K_{13}\right)} \frac{U_{0}}{U_{\mathrm{t}}^{\prime \prime}}+\left[\left(\frac{W_{\mathrm{s}} d}{2 \pi\left(K_{33}^{\prime}-K_{13}\right)} \frac{U_{0}}{U_{\mathrm{t}}^{\prime \prime}}\right)^{2}+\frac{K_{13}}{K_{33}^{\prime}-K_{13}}\right]^{1 / 2} .
$$

A first order Frederiks transitions is realized for the following values of $W_{\mathrm{s}} d$ :

$$
\begin{gathered}
W_{\mathrm{s}} d \leqslant 4\left\{\operatorname{arctg}\left[\frac{-K_{13}}{K_{11}^{\prime}+K_{13}}\right]^{1 / 2}\right\}\left[\frac{-K_{13}}{K_{11}^{\prime}+K_{13}}\right]^{1 / 2}\left(K_{11}^{\prime}+K_{13}\right) \\
W_{\mathrm{s}} d \geqslant 4\left\{\operatorname{Arth}\left[\frac{-K_{13}}{K_{33}^{\prime}-K_{13}}\right]^{1 / 2}\right\}\left[\frac{-K_{13}}{K_{33}^{\prime}-K_{13}}\right]^{1 / 2}\left(K_{33}^{\prime}-K_{13}\right) .
\end{gathered}
$$

The threshold voltages are respectively :

$$
\begin{gathered}
\left(U_{\mathrm{t}}^{\prime} / U_{0}\right)^{2} \leqslant(2 / \pi)^{2} \operatorname{arctg}^{2}\left[\frac{K_{13}}{K_{11}^{\prime}+K_{13}}\right]^{1 / 2}-\frac{2 K_{22}-K_{33}^{\prime}}{K_{11}^{\prime}}\left(\varphi_{\mathrm{m}} / \pi\right)^{2} \\
U_{\mathrm{t}}^{\prime \prime} / U_{0} \geqslant(2 / \pi) \operatorname{Arth}\left[\frac{-K_{13}}{K_{33}^{\prime}-K_{13}}\right]^{1 / 2} .
\end{gathered}
$$

As in the case $\mathrm{A}$ it is clear that when the surface parameter $W_{\mathrm{s}} d$ obeys the inequalities (19) and (20) a Frederiks transition $0-\pi / 2$ is realized in the LC. The conclusions made for homeotropic and planar layers are valid in this case as well. Spontaneous deformations are established in the twisted nematic layers when the surface energy $W_{\mathrm{s}}$ is computed from eq. (17) without the electric field. The applied electric field cause deformations $0-\pi / 2$ or $\theta_{1}-\theta_{2}-\pi / 2$ depending on the value of the surface energy.
3. Concluding remarks. - Second-order elasticity may create a first-order Frederiks transition with a hysteresis in homeotropic, planar and twisted nematic layers. The calculations show that the deformations start with a jump or a complete orientation is possible depending on the value of the surface energy and the sign of the second-order elastic coefficient $K_{13}$. For smaller values of the surface energy the LC is unstable at infinitely weak voltages. The larger values of the surface energy dictate a second order Frederiks transition.

\section{References}

[1] Rapini, A. and Papoular, M., J. Physique Colloq. 30 (1969) C4-54.

[2] Dubois-Violette, E. and De Gennes, P. G., J. Physique Lett. 36 (1975) L-155.

[3] Hinov, H. P., J. Physique Lett. 38 (1977) L-215.

[4] PIesik, B. and Koswig, H. D., II LC Conf. of Soc. Countries, Sunny Beach, Sept. 27-30 (1977) G. 3.

[5] Deuling, H. J. and Helfrich, W., Appl, Phys. Lett. 25 (1974) 129.

[6] Derzhanski, A. I. and Hinov, H. P., J. Physique 38 (1977) 1013.
[7] Rondelez, F., Thesis, Université Paris-Sud (1973),

[8] Nehring, J. and Saupe, A., J. Chem. Phys. 54 (1971) 337.

[9] Barratt, P. J. and Jenkins, J., J. Phys, A (Math. Nucl. Gen.) 6 (1973) 756.

[10] Leslie, F. M., J. Phys. D (Appl. Phys.) 3 (1970) 889.

[11] Nehring, J., Kmetz, A. R. and Schepfer, T. J., J. Appl. Phys. 47 (1976) 850.

[12] Hinov, H. P., Static deformations in NLC's, report presented at I Nat. Physics School, Gulechitsa Rila (1975).

[13] Derzhanski, A. I. and Hinov, H. P., VII Nat. Conf. on Physics, Vidin, Bulgaria, Jan. 26-30 (1976), Abstracts 133. 